Artículo original

\title{
Persistencia con estatinas en prevención primaria de enfermedad cardiovascular: resultados en una cohorte de trabajadores españoles
}

\author{
Sara Malo ${ }^{\mathrm{a}, *}$, Isabel Aguilar-Palacio ${ }^{\mathrm{a}}$, Cristina Feja ${ }^{\mathrm{a}}$, Enrica Menditto ${ }^{\mathrm{b}}$, María Jesús Lallana ${ }^{\mathrm{c}}$, \\ Elena Andrade ${ }^{\mathrm{d}}$, José Antonio Casasnovas ${ }^{\mathrm{e}}$ y María José Rabanaque ${ }^{\mathrm{a}}$ \\ a Departamento de Medicina Preventiva y Salud Pública, Universidad de Zaragoza, Fundación Instituto de Investigación Sanitaria de Aragón (IIS Aragón), Zaragoza, España \\ ${ }^{\mathrm{b}}$ Centro Interdipartimentale di Ricerca in Farmacoeconomia e Farmacoutilizzazione (CIRFF), Università degli Studi di Napoli Federico II, Nápoles, Italia \\ ' Servicio Aragonés de Salud, Instituto de Investigación Sanitaria de Aragón (IIS Aragón), Zaragoza, España \\ d Departamento de Medicina Preventiva y Salud Pública, Universidad Autónoma de Madrid/IdiPaz y CIBER de Epidemiología y Salud Pública (CIBERESP), Madrid, España \\ e Departamento de Medicina, Psiquiatría y Dermatología, Universidad de Zaragoza, Fundación Instituto de Investigación Sanitaria de Aragón (IIS Aragón), Zaragoza, España
}

Historia del artículo:

Recibido el 21 de diciembre de 2016

Aceptado el 21 de marzo de 2017

On-line el 21 de junio de 2017

\section{Palabras clave:}

Interrupción

Fármaco hipolipemiante

Farmacoepidemiología

Medicación

Medición

Enfermedad cardiovascular

Prevención primaria

\begin{abstract}
R E S U M E N
Introducción y objetivos: El objetivo de este estudio es analizar el patrón de persistencia con estatinas en prevención primaria de enfermedad cardiovascular en una cohorte de trabajadores españoles. Métodos: Este estudio descriptivo se llevó a cabo en el marco del estudio prospectivo longitudinal Aragon Workers' Health Study $(\mathrm{n}=5.400)$. Se identificó a los nuevos usuarios de estatinas varones a partir de datos recogidos en el sistema de información de consumo farmacéutico de Aragón. Se analizaron los patrones de persistencia con estatinas prescritas en prevención primaria cardiovascular, así como los potenciales predictores.

Resultados: De los 725 nuevos usuarios de estatinas, menos de un tercio habían persistido durante el año de seguimiento. Alrededor de un $15 \%$ de los usuarios no persistentes interrumpieron la terapia con estatinas tras la dispensación de la primera receta y, el 42,1\% de ellos no reiniciaron el tratamiento durante el resto del año. La mayor edad (HR = 0,55; IC95\%, 0,39-0,77) y el cotratamiento con fármacos antihipertensivos ( $\mathrm{HR}=0,68$; IC95\%, 0,56-0,82) redujeron la probabilidad de que se interrumpiera el tratamiento. No se observó asociación entre la persistencia con el tratamiento y la toma concomitante de fármacos antidiabéticos o antitrombóticos, las concentraciones basales de lipoproteínas de baja densidad o las de colesterol total. Sin embargo, la persistencia sí estuvo influida por el tipo de la primera estatina prescrita.

Conclusiones: Nuestro análisis en una cohorte de trabajadores varones sanos muestra una baja persistencia con estatinas. Estos resultados reflejan la necesidad de comprender mejor los patrones de utilización de estatinas, especialmente por individuos aparentemente sanos, y de incorporar la conducta del paciente a las decisiones de prescripción.
\end{abstract}

(c) 2017 Sociedad Española de Cardiología. Publicado por Elsevier España, S.L.U. Todos los derechos reservados.

\section{Persistence With Statins in Primary Prevention of Cardiovascular Disease: Findings From a Cohort of Spanish Workers}

A B S T R A C T

Introduction and objectives: The aim of this study was to assess patterns of treatment persistence in a cohort of male Spanish workers receiving statin therapy for primary prevention of cardiovascular disease.

Methods: This descriptive study was conducted within the framework of the prospective longitudinal Aragon Workers' Health Study $(\mathrm{N}=5400)$. Incident male statin users were identified based on data collected from the regional government's medication consumption information system. Patterns of treatment persistence with statins prescribed for primary cardiovascular disease prevention were assessed and the relevance of potential predictors explored.

Results: Among the 725 new statin users, less than one third remained persistent during the 1 year of follow-up. About 15\% of nonpersistent users discontinued statin therapy after dispensation of the first prescription; of these, $42.1 \%$ did not recommence treatment within the following year. Factors reducing the likelihood of treatment discontinuation were older age (HR, 0.55; 95\%CI, 0.39-0.77) and cotreatment with antihypertensive drugs ( $\mathrm{HR}, 0.68 ; 95 \% \mathrm{CI}, 0.56-0.82$ ). No association was observed between treatment persistence and cotreatment with antidiabetic or antithrombotic drugs, baseline low-density lipoprotein levels, or total cholesterol levels. However, persistence was influenced by the type of statin first prescribed.

VÉASE CONTENIDO RELACIONADO:

https://doi.org/10.1016/j.recesp.2017.06.032, Rev Esp Cardiol. 2018;71:4-5.

* Autor para correspondencia: Departamento de Medicina Preventiva y Salud Pública, Universidad de Zaragoza, C/ Domingo Miral s/n, 50009 Zaragoza, España.

Correo electrónico: smalo@unizar.es (S. Malo). 
Conclusions: Our analysis of a cohort of healthy male workers revealed poor statin persistence. These findings underscore the need for a better understanding of patterns of statin use, especially in apparently healthy individuals, and for the incorporation of patient behavior into prescribing decisions.

Full English text available from: www.revespcardiol.org/en

(c) 2017 Sociedad Española de Cardiología. Published by Elsevier España, S.L.U. All rights reserved.

\section{Abreviaturas}

\section{AWHS: Aragon Workers' Health Study}

cLDL: colesterol unido a lipoproteínas de baja densidad $\mathrm{CV}$ : cardiovascular

\section{INTRODUCCIÓN}

Las estatinas, al reducir el colesterol en sangre, reducen los eventos cardiovasculares (CV) y la mortalidad por cualquier causa de individuos con y sin signos de enfermedad $\mathrm{CV}^{1}$. Aunque las tasas de uso de estatinas son altas en la mayoría de las poblaciones europeas $^{2}$, el control del colesterol en dichas poblaciones es en general deficiente ${ }^{3,4}$, lo que indica que la efectividad de las estatinas puede que no corresponda exclusivamente a la eficacia demostrada en los ensayos clínicos, sino que se vea influida por los patrones de uso de estos fármacos en la práctica clínica real. Entre las características del contexto y las conductas que influyen en la consecución de los objetivos clínicos, uno de los factores clave es la "persistencia del paciente» (es decir, que el paciente persista con el tratamiento o no $)^{4-7}$. De hecho, una mayor duración del tratamiento con estatinas se asocia con una mejora de los resultados clínicos ${ }^{4-6}$.

Las tasas de persistencia en el tratamiento con estatinas en la prevención secundaria de la enfermedad CV se han evaluado en varios estudios $^{5,6,8}$. Sin embargo, hay menos información acerca de la persistencia en el caso de las estatinas usadas en prevención primaria de enfermedad CV en personas jóvenes. Tanto las pautas de tratamiento para prevención primaria como la menor edad se asocian con peor adherencia al tratamiento ${ }^{9,10}$, probablemente porque estos usuarios no se perciben «enfermos». La descripción de los patrones de persistencia en el tratamiento con estatinas en ese tipo de población es esencial para que dicha persistencia mejore $\mathrm{y}$, en última instancia, para mejorar la prevención de las condiciones crónicas.

El objetivo de este estudio es evaluar el patrón de la persistencia en el tratamiento con estatinas en prevención primaria de la enfermedad CV en una cohorte de trabajadores varones en España.

\section{MÉTODOS}

\section{Población y fuentes de datos del estudio}

El Aragon Workers' Health Study (AWHS) es un estudio prospectivo longitudinal diseñado para caracterizar los factores cardiometabólicos y evaluar la ateroesclerosis subclínica en una población de trabajadores de mediana edad del área mediterránea. Para ello, se analizaron los datos de los exámenes de salud ordinarios anuales de los trabajadores de una planta de fabricación de automóviles situada en Figueruelas, Zaragoza, que aceptaron voluntariamente participar en el estudio. El reclutamiento de la cohorte se llevó a cabo entre febrero de 2009 y mayo de 2010, con un total de 5.400 trabajadores. A los participantes de 40-55 años, se les invitó además a participar en los exámenes de diagnóstico por imagen realizados cada 3 años para evaluar la presencia de ateroesclerosis subclínica y a que respondieran a cuestionarios adicionales detallados sobre los factores CV y de estilo de vida. Al inicio del estudio, se seleccionó a 587 trabajadores (el 94,5\% varones) para que formaran parte de este grupo de seguimiento intensivo. La mayoría de los trabajadores de la cohorte realizaban trabajos de tipo manual (el 86,5\% de los varones y el 60,5\% de las mujeres). En la tabla 1 se presentan otras características basales ${ }^{11}$. La cohorte mostraba alta prevalencia de factores de riesgo CV y de ateroesclerosis subclínica, pero una prevalencia baja de enfermedad CV clínica. Se prevé continuar el seguimiento activo de los participantes hasta el año 2020. Puede consultarse información más detallada sobre el AWHS en la publicación de Casasnovas et al. ${ }^{11}$.

Este estudio descriptivo observacional se diseñó para evaluar la persistencia en el tratamiento con estatinas en la cohorte del AWHS. Los datos de prescripción de estatinas correspondientes a los participantes en el AWHS se obtuvieron de Farmasalud, el sistema de información sobre consumo de medicamentos del Gobierno de Aragón, comunidad autónoma de España donde se encuentra la fábrica de automóviles. Esta base de datos recoge la información sobre todas las prescripciones dispensadas en las farmacias de Aragón a través del sistema público de asistencia sanitaria (prescritas por un médico de la empresa o por un médico general del sistema público de asistencia sanitaria). Las prescripciones de médicos privados, compañías de seguros o el uso en pacientes hospitalizados no se recogen en Farmasalud. Cada registro en la fuente de datos corresponde a una prescripción, y contiene la siguiente información: código anónimo del paciente, sexo y fecha de nacimiento del paciente, fecha de dispensación, código ATC (Anatómico Terapéutico Químico) del fármaco prescrito, número de dosis diarias definidas y número de envases dispensados. Los fármacos se clasificaron según la versión de 2015 del código ATC de la Organización Mundial de la Salud/ sistema de dosis diarias definidas ${ }^{12}$. Usando un código encriptado proporcionado por los investigadores del AWHS, se identificaron las prescripciones de estatinas, solas (código ATC C10AA) o en combinación con otros fármacos liporreguladores (C10BA), realizadas entre el 1 de enero de 2010 y el 31 de diciembre de 2014.

A partir de la base de datos del AWHS, se obtuvieron los datos relativos a los eventos $\mathrm{CV}$ sufridos por los participantes del estudio (fecha y tipo de episodio), así como las concentraciones de

Tabla 1

Características basales en el Aragon Workers' Health Study

\begin{tabular}{lll}
\hline & Varones $(\mathrm{n}=5.048)$ & Mujeres $(\mathrm{n}=351)$ \\
\hline Edad (años) & $49,3 \pm 8,7$ & $40,8 \pm 11,6$ \\
\hline IMC & $27,7 \pm 3,6$ & $24,4 \pm 3,8$ \\
\hline PA sistólica $(\mathrm{mmHg})$ & $127,0 \pm 14,7$ & $111,4 \pm 13,2$ \\
\hline PA diastólica $(\mathrm{mmHg})$ & $83,8 \pm 10,1$ & $76,4 \pm 9,5$ \\
\hline Colesterol total $(\mathrm{mg} / \mathrm{dl})$ & $212,4 \pm 37,6$ & $204,3 \pm 39,9$ \\
\hline cHDL $(\mathrm{mg} / \mathrm{dl})$ & $52,4 \pm 11,0$ & $66,5 \pm 14,2$ \\
\hline Triglicéridos $(\mathrm{mg} / \mathrm{dl})$ & $148,8 \pm 106,1$ & $89,7 \pm 75,5$ \\
\hline Glucosa $(\mathrm{mg} / \mathrm{dl})$ & $98,3 \pm 19,6$ & $91,2 \pm 16,3$ \\
\hline Tabaquismo & & \\
\hline Nunca han fumado & $1.796(35,8)$ & $146(41,8)$ \\
\hline Exfumadores & $1.359(27,1)$ & $46(13,2)$ \\
\hline Fumador actual & $1.862(37,1)$ & $157(45,0)$ \\
\hline
\end{tabular}

cHDL: colesterol unido a lipoproteínas de alta densidad; IMC: índice de masa corporal; PA: presión arterial.

Los datos expresan $\mathrm{n}(\%)$ o media \pm desviación estándar.

Reproducido con permiso de Casasnovas et al. ${ }^{11}$. 
colesterol total y otras fracciones lipídicas registradas en los exámenes médicos anuales. El colesterol unido a lipoproteínas de baja densidad (cLDL) de cada paciente en cada examen se calculó con la fórmula de Friedewald ${ }^{13}$, basada en el colesterol total, el colesterol unido a lipoproteínas de alta densidad y los triglicéridos.

En cada paciente, la fecha de la primera dispensación de una estatina se definió como fecha índice para el estudio. Los análisis se limitaron a los nuevos usuarios de estatinas, definidos como los que no habían recibido ninguna prescripción de estatinas en los 12 meses previos a la fecha índice, entre el 1 de enero de 2011 y el 31 de diciembre de 2013. Dada su baja frecuencia en la población del AWHS, se excluyó de los análisis a las mujeres $(4,2 \%)$ y a los varones que habían sufrido un evento CV antes del inicio del tratamiento con estatinas (prevención secundaria, 3,9\%).

\section{Evaluación de la persistencia}

La persistencia se definió como la continuidad del tratamiento durante un seguimiento de 1 año a partir de la fecha índice. La determinación de la persistencia se basó en el tiempo (intervalo) transcurrido entre una dispensación de un fármaco y la siguiente. Por este motivo, se excluyó del análisis a los participantes que recibieron solo 1 prescripción de estatinas durante el periodo de estudio o que recibieron 1 o más prescripciones únicamente en la fecha índice. Se consideró que un participante no presentaba persistencia si el intervalo entre 2 dispensaciones sucesivas era mayor que el doble de la duración de la prescripción precedente. Aunque otros autores han definido este intervalo empleando un número de días fijo, en este estudio se optó por otro enfoque, dado que la ventana de tiempo entre las dispensaciones es variable (1-2 meses) en la comunidad autónoma de Aragón. El número de días de medicación proporcionado se estimó a partir del número de comprimidos y envases. En España, la mayor parte de las estatinas se venden en envases de 28 comprimidos, con la excepción de algunas presentaciones de lovastatina, pravastatina/fenofibrato $\mathrm{y}$ atorvastatina/ezetimiba, que se venden en envases de $30 \mathrm{com}$ primidos. Del total de prescripciones dispensadas a los participantes en el AWHS, solo 2 correspondieron a lovastatina. La posología prescrita de las estatinas es de 1 comprimido al día. En consecuencia, partiendo del supuesto de que cada envase contenía 28 dosis diarias, el intervalo máximo aceptado fue de 56 días. No se tuvo en cuenta la acumulación del aporte de medicación con el paso del tiempo (es decir, solo se utilizó el número de comprimidos de la última prescripción dispensada para evaluar el intervalo de tiempo). Se censuró a los participantes en el análisis si superaban el intervalo aceptado sin adquirir una nueva prescripción, o al llegar al final del periodo de estudio (en el caso de que hubiera habido persistencia durante todo el periodo de seguimiento). Se clasificó a los participantes no persistentes como usuarios que reiniciaban un tratamiento con estatinas después de un periodo de interrupción o como usuarios que simplemente interrumpían el tratamiento (y no recibían ninguna prescripción de estatina después del intervalo máximo aceptable).

\section{Análisis estadístico}

Se registraron las características de los usuarios de estatinas que iniciaron tratamiento. La edad en la fecha índice se clasificó de la siguiente manera: $<50,50-54,55-59$ y $\geq 60$ años. La determinación del tratamiento antidiabético, antihipertensivo o antitrombótico concomitante se evaluó teniendo en cuenta la existencia de cualquier prescripción para cada uno de los respectivos procesos, durante el periodo transcurrido entre la fecha índice de la estatina y la de interrupción o final del seguimiento. Los valores basales de colesterol total y cLDL registrados en el último examen médico del paciente anterior a la fecha índice se clasificaron como $\operatorname{cLDL}<100,100-154,155-189$ $\mathrm{y} \geq 190$, y colesterol total $<200,200-240 \mathrm{y}>240 \mathrm{mg} / \mathrm{dl}$. Estas categorías se establecieron según los valores de referencia proporcionados por la guía europea para la prevención de la enfermedad $\mathrm{CV}^{14}$ en individuos con riesgo CV bajo-moderado, como los que formaban parte de la población del presente estudio.

Las tasas de persistencia se analizaron longitudinalmente con el método de Kaplan-Meier. Las diferencias estadísticas entre las curvas se evaluaron mediante test de $\log$ rank. La probabilidad de falta de persistencia durante el seguimiento se determinó con un análisis multivariablee mediante regresión de Cox. Se calcularon los valores de hazard ratio (HR) y los intervalos de confianza del 95\% (IC95\%) ajustando por grupo de edad en la fecha índice, el tratamiento concomitante antidiabético, antihipertensivo o antitrombótico y los valores basales de cLDL y colesterol total.

Se llevó a cabo un análisis de sensibilidad para evaluar la posible influencia de la duración del intervalo elegida en la clasificación de los participantes. Se recalculó la proporción de participantes persistentes con estatinas aplicando duraciones del intervalo de 1,5 y 2,5 veces el número de dosis diarias (es decir, 42 y 70 días respectivamente).

Todos los análisis se realizaron con el programa STATA versión 12.1 (StataCorp; College Station, Texas, Estados Unidos).

\section{Ética}

Los participantes en el AWHS aportaron su consentimiento informado por escrito cuando se incorporaron al estudio, y la investigación que se presenta aquí está autorizada por el Comité de Ética de Investigación Clínica de Aragón.

\section{RESULTADOS}

\section{Características de los nuevos usuarios de estatinas}

Entre los 5.400 participantes del AWHS, 725 nuevos usuarios de estatinas cumplían los criterios de inclusión (figura 1), con una media \pm desviación estándar de edad de 54,7 \pm 5,1 años. La clasificación por edades mostró el siguiente desglose: $<50$ años, $13,1 \%$; 50-54, 31,9\%; 55-59, 36,8\%, y $\geq 60,18,2 \%$.

La mediana (intervalo) de valores basales de cLDL fue 159,6 $(58,0-294,8) \mathrm{mg} / \mathrm{dl}$. La distribución de los usuarios de estatinas en las diferentes categorías de cLDL fue: $<100 \mathrm{mg} / \mathrm{dl}, 26(3,8 \%)$; $100-154 \mathrm{mg} / \mathrm{dl}, 273$ (39,7\%); 155-189 mg/dl, 294 (42,8\%), y $\geq 190 \mathrm{mg} / \mathrm{dl}, 94$ (13,7\%). La mediana (intervalo) de valores de colesterol total fue $247,0(142,0-481,0) \mathrm{mg} / \mathrm{dl}$, y la distribución de los usuarios de estatinas fue: $<200 \mathrm{mg} / \mathrm{dl}, 85$ (11,8\%); $200-240 \mathrm{mg} / \mathrm{dl}$, 213 (29,5\%), y > $240 \mathrm{mg} / \mathrm{dl}, 425$ (58,8\%). La mediana (intervalo) de otros factores de riesgo CV fue: glucemia, $98,0(61,0-292,0) \mathrm{mg} / \mathrm{dl}$; presión arterial diastólica, $86,0(60,0-129,0) \mathrm{mmHg}$; presión arterial sistólica, 128,0 (90,0-220,0) mmHg, e índice de masa corporal, 27,8 $(19,3-43,6)$.

Durante el periodo de seguimiento correspondiente, el 49,8\% de los usuarios de estatinas recibieron tratamiento junto con otros fármacos para la prevención de la enfermedad CV: el 36,3\% con 1 fármaco adicional, el $11,7 \%$ con 2 y el $1,8 \%$ con 3 . Concretamente, el $41,4 \%$ estaba tomando antihipertensivos; el 12,3\%, antitrombóticos y el 11,5\%, antidiabéticos.

La estatina prescrita con más frecuencia en la fecha índice fue la simvastatina (el $40,4 \%$ de los nuevos usuarios), seguida de atorvastatina $(24,7 \%)$, rosuvastatina $(24,4 \%)$, pitavastatina $(5,8 \%)$, pravastatina $(2,3 \%)$, simvastatina/ezetimiba $(1,4 \%)$, fluvastatina $(0,8 \%)$ y lovastatina $(0,1 \%)$.

El 22,6\% de las prescripciones de estatinas las hizo un médico de la empresa y el $77,4 \%$, un médico general del sistema público de asistencia sanitaria. 


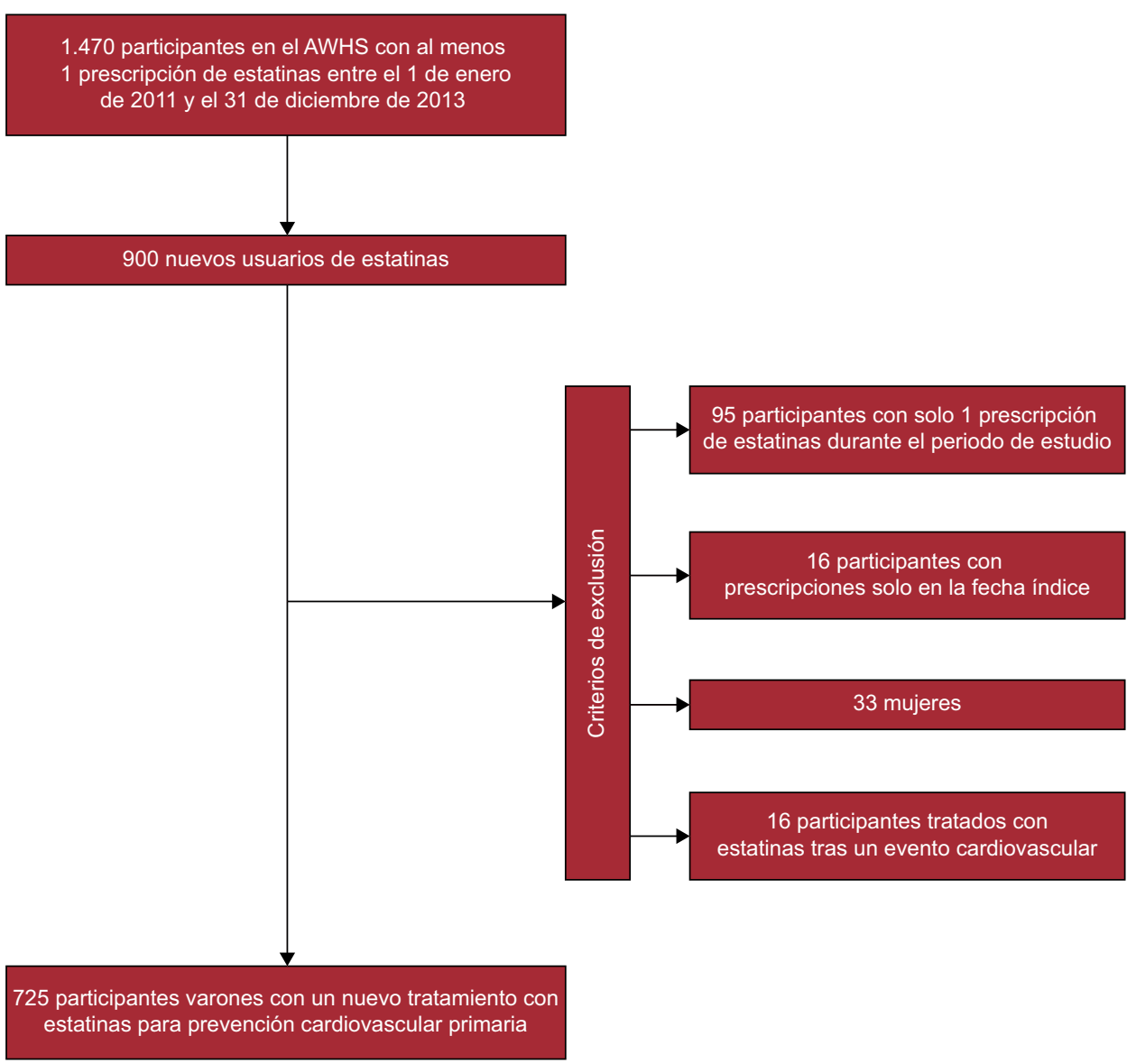

Figura 1. Diagrama de flujo de la población del estudio. AWHS: Aragon Workers' Health Study.

\section{Evaluación de la persistencia}

De los 725 nuevos usuarios de estatinas, el 29,5\% continuó con el tratamiento durante el seguimiento de 1 año. La media de tiempo transcurrido entre la fecha índice y la interrupción del tratamiento fue 211,3 días. Según el análisis de Kaplan-Meier, la mitad del total de nuevos usuarios de estatinas mostraba persistencia con el tratamiento a los 184 días de la fecha índice y el 36,9\%, a los 270 días. La curva de supervivencia mostró una caída pronunciada a

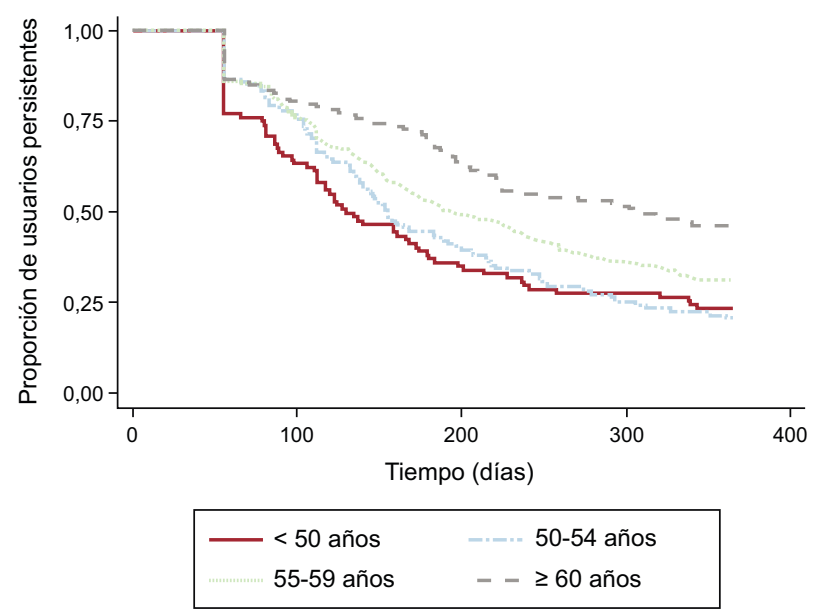

Figura 2. Persistencia con estatinas en prevención primaria de la enfermedad cardiovascular entre los participantes varones del Aragon Workers' Health Study, por grupos de edad. los 56 días, el final del primer intervalo aceptable (28 dosis diarias $\times 2$ ), lo que indica que alrededor de un $15 \%$ de los nuevos usuarios de estatinas interrumpieron el tratamiento inmediatamente después de la primera prescripción. De los pacientes que interrumpieron el tratamiento, el 57,9\% reinició más tarde el tratamiento con una estatina, mientras que al $42,1 \%$ no se le dispensó ninguna otra estatina durante el seguimiento.

Se observaron diferencias en las tasas de persistencia según el grupo de edad (test de log rank, $\mathrm{p}<0,0001$ ) (figura 2), que aumentan con la edad. El análisis de las tasas de persistencia correspondientes a las 3 estatinas prescritas con más frecuencia reveló unos valores más altos para los nuevos usuarios de simvastatina que para los de rosuvastatina o atorvastatina (test de $\log \operatorname{rank}, \mathrm{p}<0,05$ ).

\section{Factores asociados con la persistencia}

En el análisis bivariable, la HR bruta para la interrupción del tratamiento con estatinas (tabla 2) en los 2 grupos de más edad (55-59 y $\geq 60$ años) fue significativamente menor que en el grupo de menos edad ( $<50$ años), y en los individuos que recibían tratamientos antihipertensivos concomitantes también fue menor que entre quienes no recibían otros fármacos para la prevención de enfermedad CV. En cambio, se observaron valores de HR más altos en los participantes con un valor basal del colesterol total $>240 \mathrm{mg} / \mathrm{dl}$ que en quienes lo tenían $<200 \mathrm{mg} / \mathrm{dl}$; también en los participantes con cLDL basal $\geq 190 \mathrm{mg} / \mathrm{dl}$ en comparación con los que lo tenían $<100 \mathrm{mg} /$ dl. El análisis multivariable (tabla 2) mostró una HR estadísticamente significativa solo para los usuarios de estatinas del grupo de más edad ( $\geq 60$ años) (HR = 0,55; IC95\%, 0,39-0,77) y los que recibían tratamiento concomitante con fármacos antihipertensivos (HR = 0,68; IC95\%, 0,56-0,82). 
Tabla 2

Valores de hazard ratio de la interrupción del tratamiento con estatinas en una cohorte de trabajadores varones de España sin eventos cardiovasculares previos. El tratamiento con estatinas se inició entre el 1 de enero de 2011 y el 31 de diciembre de 2013

\begin{tabular}{|c|c|c|}
\hline & HR (IC95\%) & HRa (IC95\%) \\
\hline \multicolumn{3}{|l|}{ Grupo de edad } \\
\hline$<50$ años & 1 & 1 \\
\hline 50-54 años & $0,95(0,72-1,24)$ & $0,98(0,74-1,31)$ \\
\hline 55-59 años & $0,73(0,56-0,96)^{\mathrm{c}}$ & $0,79(0,59-1,05)$ \\
\hline$\geq 60$ años & $0,49(0,35-0,68)^{\mathrm{c}}$ & $0,55(0,39-0,77)^{c}$ \\
\hline Uso concomitante de antidiabéticos ${ }^{\mathrm{a}}$ & $0,83(0,63-1,11)$ & $1,12(0,83-1,52)$ \\
\hline Uso concomitante de antihipertensivos ${ }^{\mathrm{a}}$ & $0,64(0,53-0,76)^{c}$ & $0,68(0,56-0,82)^{\mathrm{c}}$ \\
\hline Uso concomitante de antitrombóticos ${ }^{\mathrm{a}}$ & $0,81(0,61-1,06)$ & $0,97(0,72-1,32)$ \\
\hline \multicolumn{3}{|l|}{$c L D L$ basal $^{\mathrm{b}}$} \\
\hline$<100 \mathrm{mg} / \mathrm{dl}$ & 1 & 1 \\
\hline $100-154 \mathrm{mg} / \mathrm{dl}$ & $1,66(0,96-2,85)$ & $1,59(0,86-2,91)$ \\
\hline $155-189 \mathrm{mg} / \mathrm{dl}$ & $1,70(0,99-2,92)$ & $1,38(0,71-2,68)$ \\
\hline$\geq 190 \mathrm{mg} / \mathrm{dl}$ & $1,96(1,10-3,48)^{c}$ & $1,46(0,73-2,92)$ \\
\hline \multicolumn{3}{|l|}{ Colesterol total basal } \\
\hline$<200 \mathrm{mg} / \mathrm{dl}$ & 1 & 1 \\
\hline $200-240 \mathrm{mg} / \mathrm{dl}$ & $1,31(0,95-1,79)$ & $1,13(0,79-1,63)$ \\
\hline$>240 \mathrm{mg} / \mathrm{dl}$ & $1,42(1,06-1,91)^{\mathrm{c}}$ & $1,26(0,83-1,91)$ \\
\hline
\end{tabular}

cLDL: colesterol unido a lipoproteínas de baja densidad; HR: hazard ratio; HRa: HR ajustada; IC95\%: intervalo de confianza del 95\%.

En todos los análisis excepto el del cLDL, $\mathrm{n}=725$. En el análisis bivariable del cLDL y modelo de regresión multivariable, se incluyó a 687 participantes.

a Uso no concomitante como referencia.

b Valores de C-LDL y de colesterol total registrados en el último examen médico antes del inicio del tratamiento con estatinas.

Resultado estadísticamente significativo.

\section{Análisis de sensibilidad}

Las proporciones de nuevos usuarios de estatinas persistentes con el tratamiento fueron del 13,2 y el $42,8 \%$ según se aplicaran los intervalos de 1,5 y 2,5 veces el número de dosis diarias dispensadas respectivamente. La aplicación de estos intervalos diferentes confirmó los resultados del análisis de regresión de Cox (datos no presentados).

\section{DISCUSIÓN}

El análisis de una cohorte de trabajadores varones mostró que menos de un tercio de los nuevos usuarios de estatinas persistían en el tratamiento durante el primer año tras la prescripción inicial. La probabilidad de persistencia con estatinas aumenta con la edad. En un metanálisis previo de estudios que evaluaban los factores predictores de la falta de adherencia a las estatinas ${ }^{15}$, se observó una menor probabilidad de persistencia y adherencia en los usuarios de estatinas más jóvenes ( $<50$ años) y los más ancianos ( $>70$ años). Aunque los resultados del presente estudio confirmaron la asociación entre la baja persistencia en el tratamiento y la edad menor, no se pudo evaluar el patrón en los individuos de más de 70 años, que no estaban representados en la cohorte del AWHS.

La mitad de los nuevos usuarios de estatinas de la población del estudio estaban tomando al menos 1 tratamiento adicional para la prevención de la enfermedad CV, y, de estos, el 41,4\% recibía un tratamiento antihipertensivo concomitante. La probabilidad de persistencia fue mayor en estos participantes, lo cual es coherente con lo indicado por estudios previos ${ }^{16,17}$. Sin embargo, esos mismos estudios identificaron también que ciertos tratamientos concomitantes para otros factores de riesgo $\mathrm{CV}$, como los fármacos antidiabéticos o antitrombóticos, eran factores predictores de la continuidad del tratamiento con estatinas. Además de la influencia conocida de factores como la edad del paciente o la situación socioeconómica ${ }^{16,17}$, la presencia de otros factores de riesgo CV puede influir en el tratamiento a largo plazo en los individuos que no sufren enfermedad $\mathrm{CV}$, probablemente porque esos pacientes perciben mayor riesgo de enfermedad. Según algunos autores, esta asociación no es aplicable a los adultos de más edad, en cuya adherencia a las estatinas influyen también otros factores como la complejidad de la pauta de administración, la mayor incidencia de acontecimientos adversos y el deterioro $\operatorname{cognitivo~}^{15}$. Por otra parte, se ha planteado la hipótesis de que los individuos a los que su médico ha advertido de los riesgos de las altas concentraciones de colesterol son más conscientes de la importancia del control de la enfermedad CV y, por lo tanto, pueden tener una mayor adherencia al tratamiento ${ }^{8}$. Según nuestro conocimiento, solo 1 estudio previo, realizado en una población sueca $^{8}$, ha analizado la posible asociación entre los valores basales de colesterol y la persistencia en el tratamiento. Los autores indicaron que los valores basales de cLDL altos se asociaban de manera significativa con una menor tasa de interrupción del tratamiento en los pacientes no diabéticos sin eventos CV previos. En cambio, en el presente estudio no se observó una asociación clara entre los valores basales de colesterol y la persistencia en el tratamiento tras haber ajustado por otras variables.

La simvastatina es la estatina prescrita con más frecuencia en la cohorte de estudio (al 40,4\% de los nuevos usuarios de estatinas). Según las actuales recomendaciones europeas ${ }^{14}$, el tipo de estatina prescrito debe elegirse en función del riesgo $\mathrm{CV}$ total del paciente y la reducción porcentual de cLDL esperada, que depende del valor basal. La prescripción frecuente de una estatina de baja potencia como fármaco inicial parece razonable, dado que los trabajadores de esta cohorte de estudio eran, en su mayoría, individuos sanos, con medianas de cLDL y colesterol total consideradas moderadasaltas, y menos de un $15 \%$ tenía 2 o más factores de riesgo CV concomitantes. Los resultados indicaron también que los usuarios de simvastatina tenían una probabilidad de persistir en el tratamiento significativamente superior a la de los tratados con estatinas de mayor potencia, como la atorvastatina y la rosuvastatina. De igual modo, en su estudio de una población de mediana edad sin enfermedad CV de Quebec (Canadá), Perreault et al. ${ }^{16}$ describieron un aumento del riesgo de falta de persistencia en los pacientes a los que se prescribió atorvastatina, en comparación con los tratados con simvastatina o pravastatina. Por el contrario, en un amplio estudio de base poblacional realizado en usuarios de estatinas para la prevención de la enfermedad CV primaria ${ }^{17}$ en Reino Unido, no se observó asociación alguna entre el tipo de estatina prescrito y el riesgo de interrupción del tratamiento. Resulta difícil llegar a una conclusión general basada en los resultados de múltiples estudios de factores predictores de la persistencia debido a la variabilidad de las poblaciones estudiadas, los datos disponibles y los métodos empleados. Haber excluido del análisis a las mujeres limita la comparabilidad de los resultados, en especial teniendo en cuenta la asociación demostrada entre sexo y adherencia y persistencia ${ }^{9,15-17}$. Por otra parte, es probable que el trabajo en una fábrica se asociara con diversas características sociales, médicas y de utilización de servicios sanitarios que influyeran en la conducta del paciente ${ }^{15}$, que pueden diferir de las que se dan en la población general.

Se observó que alrededor del 15\% de los nuevos usuarios de estatinas interrumpieron el tratamiento inmediatamente tras la compra de la primera prescripción. Se estima que casi un $12 \%$ de los usuarios de estatinas sufren efectos adversos atribuidos a estos fármacos ${ }^{7}$, lo que puede explicar por qué estos pacientes interrumpieron precozmente el tratamiento $\mathrm{y}$ se negaron a reanudarlo. De hecho, los resultados indican que el $42,1 \%$ del total de usuarios clasificados como individuos sin persistencia no 
reiniciaron el tratamiento con estatinas durante el seguimiento de 1 año. Esta observación concuerda con los resultados del estudio en población de Reino Unido antes mencionado ${ }^{17}$, en el que un $45 \%$ de los usuarios de estatinas en prevención primaria interrumpieron el tratamiento y no lo reiniciaron durante el año siguiente. Los autores de ese estudio señalaron que el sexo femenino, la mayor edad, el índice de masa corporal inferior y la demencia se asocian con mayor riesgo de interrupción y menor probabilidad de reiniciar el tratamiento con estatinas.

\section{Limitaciones}

El presente estudio tiene varias limitaciones. Las principales corresponden a la fuente de información utilizada. Dado que la base de datos Farmasalud registra únicamente las prescripciones realizadas a través el sistema público de asistencia sanitaria, es posible que la persistencia no se haya estimado adecuadamente en algunos casos. Sin embargo, es improbable que hubiera participantes que pasaran de un médico del servicio público de asistencia sanitaria a un médico privado. Los participantes en el AWHS tienen fácil acceso a un médico cuya consulta está situada cerca de su área de trabajo. En consecuencia, los trabajadores de la empresa suelen acudir al mismo médico. Otra posibilidad es que consulten a un médico general perteneciente al sistema de asistencia sanitaria de financiación pública, en cuyo caso los datos de prescripción se habrán registrado en la misma fuente de datos.

En la fuente de datos tampoco constaban la dosis prescrita y la cantidad de fármaco dispensada (solo se dispuso del número de dosis diarias definidas). Dadas las conocidas limitaciones de la dosis diaria definida como unidad de medida ${ }^{18}$, se convirtió el número de comprimidos en la dosis diaria basándose en las presentaciones comercializadas de las estatinas. En nuestra opinión, este enfoque es válido cuando la dosis recomendada es siempre igual a un número de comprimidos fijo.

La fuente de datos del presente estudio aportó información sobre un pequeño número de variables relativas a los participantes y sus pautas de tratamiento. No se dispuso de información sobre el motivo de iniciar el tratamiento, los antecedentes familiares, las características clínicas, las prescripciones de tratamientos distintos de los indicados para la prevención de enfermedad CV, las características de los médicos o la actitud de los pacientes, lo que habría tenido interés para identificar otros factores predictores de la persistencia en el tratamiento. La falta de información respecto al motivo de la prescripción es una limitación determinante para la validez del estudio y se debe principalmente a que la fuente de datos no se diseñó para ese fin.

Una limitación frecuente de los análisis de datos de dispensación de fármacos es el supuesto de que la compra de un medicamento equivale a su consumo. El objetivo es determinar las tasas de persistencia, que pueden ser un indicador de la adherencia al tratamiento más apropiado que la adherencia en sí, dado que se basa en la continuidad en la recogida de las prescripciones, y no en el número de dosis disponibles. Que una persona acuda regularmente a la farmacia a adquirir su medicación es un probable indicador de que está tomando la medicación prescrita.

Además de las limitaciones antes mencionadas en relación con la fuente de datos, la variedad de metodologías empleadas para definir y evaluar la persistencia en el tratamiento y la falta de métodos estandarizados complican el cálculo y la comparación de los resultados. Se observa una considerable variabilidad, por ejemplo, en la duración máxima aceptable entre las dispensaciones. En 2 estudios de poblaciones en Suecia ${ }^{8}$ y Reino Unido ${ }^{17}$, se evaluó la persistencia en el tratamiento entre nuevos usuarios de estatinas aplicando un intervalo de 90 días tras la fecha de la última dispensación del medicamento prescrito. Los autores del estudio de Suecia basaron esta decisión en los reglamentos nacionales, que establecen una ventana temporal de hasta 3 meses entre las sucesivas dispensaciones. Tal como se preveía, ambos estudios indicaron unas tasas de persistencia con estatinas (el 68 y el 53\% respectivamente) mayores que las descritas aquí (29,5\%). Aunque inicialmente no se consideró el intervalo de 2,5 veces la dosis diaria como el más apropiado para evaluar la persistencia en el tratamiento, el porcentaje de usuarios con persistencia obtenido con este valor (el 42,8\% de los nuevos usuarios de estatinas) pareció más realista y coherente con lo observado en las demás poblaciones. Aun cuando los análisis de sensibilidad respaldaron la solidez de los resultados y la duración del intervalo aplicadas, debe señalarse que, para asegurar la comparabilidad, son esenciales métodos y criterios de cálculo de los indicadores de la persistencia claramente definidos. Hay varias situaciones frecuentes que contribuyen a que se subestime la persistencia en el tratamiento con estatinas. Por ejemplo, se puede clasificar erróneamente a algunos participantes como sin persistencia, cuando en realidad su tratamiento se ha cambiado de una estatina a un fármaco hipolipemiante distinto de las estatinas. Sin embargo, esta situación es improbable, ya que las estatinas, solas o en combinación, están recomendadas como primera línea de tratamiento para la hipercolesterolemia ${ }^{14}$. Asimismo un usuario podría adquirir el primer envase de estatinas tras la consulta médica, pero esperar varios días antes de iniciar el tratamiento, con lo cual se superaría el intervalo aceptado y se produciría una clasificación errónea al considerar que ha habido una interrupción.

Por último, formaron la población del estudio únicamente varones sanos y relativamente jóvenes, lo cual dificulta la extrapolación de los resultados a la población general de nuevos usuarios de estatinas. La población del estudio corresponde a una cohorte de voluntarios que son especialmente conscientes del riesgo CV y se someten a revisiones y análisis clínicos anuales, a la vez que reciben recomendaciones de salud regulares y detalladas. Se sospecha que la persistencia en el tratamiento con estatinas para la prevención primaria de las enfermedades CV en contextos de asistencia sanitaria distintos del AWHS podría ser aún más baja.

No obstante, estos resultados son una contribución importante al conocimiento de los patrones de uso de medicamentos para la prevención de la enfermedad CV, incluidas las estatinas. La prescripción de estatinas en poblaciones como la descrita aquí continúa siendo objeto de controversia. Estos fármacos están recomendados por la guía clínica actual ${ }^{14}$ como tratamiento de primera línea para los pacientes con un riesgo $\mathrm{CV}$ elevado e hipercolesterolemia (no familiar) o una hiperlipemia combinada, y son de fácil acceso por su bajo coste. Sin embargo, la evidencia existente indica que las estatinas de alta potencia se asocian con mayor riesgo de efectos adversos, como la diabetes de nueva aparición, en especial para los pacientes con riesgo de sufrir diabetes $^{19}$. Además, algunos autores ${ }^{20}$ han descrito que las evaluaciones económicas de estos fármacos patrocinadas por la industria farmacéutica, en especial de las estatinas utilizadas para la prevención primaria de la enfermedad $\mathrm{CV}$, suelen ser favorables al perfil de coste-efectividad de los productos en cuestión.

De todos modos, es innegable la importancia de la conducta del paciente en el tratamiento de los factores de riesgo $\mathrm{CV}$. Una revisión sistemática ${ }^{6}$ de los efectos en los resultados clínicos de la adherencia y la persistencia en el tratamiento con estatinas ha indicado que, en prevención primaria, se observaron beneficios clínicos en eventos CV tras el primer año de tratamiento continuo. Esto subraya la importancia del compromiso del paciente y de su participación en el control de la enfermedad CV, así como la necesidad de una mayor evaluación de las repercusiones del uso persistente de estatinas en las concentraciones de colesterol y, por consiguiente, en el riesgo de enfermedad CV. Además, debe tenerse en cuenta que el control de la enfermedad CV debe realizarse mediante un control conjunto de todos los factores de riesgo, y no 
solo mediante el tratamiento farmacológico, sino también con cambios en el estilo de vida ${ }^{21}$. Por último, para los pacientes que no alcanzan los objetivos recomendados, hay que considerar otras alternativas terapéuticas ${ }^{22}$.

\section{CONCLUSIONES}

Los resultados de este estudio ponen de manifiesto una baja continuidad o persistencia con el tratamiento en los nuevos usuarios de estatinas de una población de trabajadores varones sanos de España. Se observa que una parte significativa de la población del estudio interrumpió el tratamiento poco después de iniciarlo, y que el tipo de estatina prescrito influye en la probabilidad de persistencia. El tratamiento concomitante con fármacos antihipertensivos y la mayor edad han resultado factores predictivos de una mayor persistencia con el tratamiento.

En el contexto de la prevención primaria de la enfermedad CV, serán necesarios más estudios observacionales de utilización de medicamentos para ampliar el conocimiento de los patrones de uso de las estatinas y comprender mejor el efecto que las tasas de persistencia individuales tienen en los resultados clínicos. Esto sería un punto de partida útil para ajustar las recomendaciones de prescripción cuando las tasas de adherencia y persistencia son insuficientes $\mathrm{y}$, por consiguiente, no se alcanzan los objetivos lipídicos.

\section{FINANCIACIÓN}

El AWHS cuenta con el apoyo y la financiación de un contrato firmado en 2009 entre el Gobierno de Aragón (IACS [Instituto Aragonés de Ciencias de la Salud]) y el Fondo de Investigaciones Sanitarias del Ministerio de Salud de España (CNIC [Centro Nacional de Investigación Cardiovascular]). Este estudio contó con financiación externa del Proyecto del Fondo de Investigación Sanitaria, Instituto de Salud Carlos III y el fondo Europeo de Desarrollo Regional (FEDER) (PI13/01668).

\section{CONFLICTO DE INTERESES}

No se declara ninguno.

\section{¿QUÉ SE SABE DEL TEMA?}

- Para que aporten un tratamiento efectivo, la toma de estatinas debe ser continua. Sin embargo, las tasas de persistencia en el tratamiento con estos fármacos son inferiores a lo esperado, sobre todo en poblaciones con riesgo CV bajo.

- Las causas de la falta de persistencia son multifactoriales.

\section{¿QUÉ APORTA DE NUEVO?}

- Este estudio describe mala persistencia con estatinas en una cohorte de trabajadores varones sanos.

- Una gran proporción de los nuevos usuarios de estatinas interrumpieron el tratamiento inmediatamente después de iniciarlo, y en numerosos casos no lo reiniciaron durante el año siguiente.

- Aunque se han identificado algunos factores asociados con la mala persistencia, se necesita mejor conocimiento de los determinantes de la falta de persistencia que influyen directamente en los resultados de salud finales.

\section{BIBLIOGRAFÍA}

1. Taylor F, Huffman MD, Macedo AF, et al. Statins for the primary prevention of cardiovascular disease. Cochrane Database Syst Rev. 2013;1:CD004816.

2. Agencia Española de Medicamentos y Productos Sanitarios. Utilización de medicamentos hipolipemiantes en España durante el periodo 2000-2012. 2014 [consultado 8 Mar 2016]. Disponible en: http://www.aemps.gob.es/ medicamentosUsoHumano/observatorio/docs/hipolipemiantes-2000-2012.pdf.

3. Guallar-Castillon P, Gil-Montero M, Leon-Munoz LM, et al. Magnitude and Management of Hypercholesterolemia in the Adult population of Spain, 2008-2010: The ENRICA Study. Rev Esp Cardiol. 2012;65:551-558.

4. Garcia-Gil M, Blanch J, Comas-Cufi M, et al. Patterns of statin use and cholesterol goal attainment in a high-risk cardiovascular population: A retrospective study of primary care electronic medical records. J Clin Lipidol. 2016;10:134-142.

5. De Vera MA, Bhole V, Burns LC, et al. Impact of statin adherence on cardiovascular disease and mortality outcomes: a systematic review. Br J Clin Pharmacol. 2014;78:684-698

6. Simpson Jr RJ, Mendys P. The effects of adherence and persistence on clinical outcomes in patients treated with statins: a systematic review. J Clin Lipidol. 2010;4:462-471

7. Spence JD, Dresser GK. Overcoming Challenges With Statin Therapy. J Am Heart Assoc. 2016;5:

8. Citarella A, Linder M, Kieler H, et al. Influence of baseline low-density lipoprotein cholesterol values on statin therapy persistence. Eur J Clin Pharmacol. 2016;72:349-357.

9. Ferrajolo C, Arcoraci V, Sullo MG, et al. Pattern of statin use in southern italian primary care: can prescription databases be used for monitoring long-term adherence to the treatment? PLoS One. 2014;9:e102146.

10. Yeaw J, Benner JS, Walt JG, et al. Comparing adherence and persistence across 6 chronic medication classes. J Manag Care Pharm. 2009;15:728-740.

11. Casasnovas JA, Alcaide V, Civeira F, et al. Aragon workers' health study-design and cohort description. BMC Cardiovasc Disord. 2012;12:45.

12. World Health Organization Collaborating Centre for Drug Statistics Methodology. ATC/DDD Index Oslo, Norway: Norwegian Institute of Public Health; 2017 [consultado 20 Mar 2016]. Disponible en: http://www.whocc.no/atc_ddd_index/.

13. Friedewald WT, Levy RI, Fredrickson DS. Estimation of the concentration of lowdensity lipoprotein cholesterol in plasma, without use of the preparative ultracentrifuge. Clin Chem. 1972;1:499-502.

14. Piepoli MF, Hoes AW, Agewall S, et al. 2016 European Guidelines on cardiovascular disease prevention in clinical practice: The Sixth Joint Task Force of the European Society of Cardiology and Other Societies on Cardiovascular Disease Prevention in Clinical Practice (constituted by representatives of 10 societies and by invited experts): Developed with the special contribution of the European Association for Cardiovascular Prevention \& Rehabilitation (EACPR). Eur Heart J. 2016;37:2315 2381.

15. Mann DM, Woodward M, Muntner P, et al. Predictors of nonadherence to statins: a systematic review and meta-analysis. Ann Pharmacother. 2010:44:1410-1421.

16. Perreault S, Blais L, Dragomir A, et al. Persistence and determinants of statin therapy among middle-aged patients free of cardiovascular disease. Eur J Clin Pharmacol. 2005;61:667-674.

17. Vinogradova Y, Coupland C, Brindle P, et al. Discontinuation and restarting in patients on statin treatment: prospective open cohort study using a primary care database. BMJ. 2016;353:i3305.

18. Sinnott SJ, Polinski JM, Byrne $S$, et al. Measuring drug exposure: concordance between defined daily dose and days' supply depended on drug class. J Clin Epidemiol. 2016;69:107-113.

19. Agouridis AP, Kostapanos MS, Elisaf MS. Statins and their increased risk of inducing diabetes. Expert Opin Drug Saf. 2015;14:1835-1844.

20. Catala-Lopez F, Sanfelix-Gimeno G, Ridao M, et al. When are statins cost-effective in cardiovascular prevention?. A systematic review of sponsorship bias and conclusions in economic evaluations of statins. PLoS One. 2013;8:e69462.

21. Castro Conde A, Marzal Martin D, Dalmau Gonzalez-Gallarza R, et al. Vascular Risk and Cardiac Rehabilitation 2015: A Selection of Topical Issues. Rev Esp Cardiol. 2016;69:294-299.

22. Anguita Sanchez M, Castro Conde A, Cordero Fort A, et al. Challenges in Oral Lipidlowering Therapy: Position Document of the Spanish Society of Cardiology. Rev Esp Cardiol. 2016;69:1083-1087. 